\title{
The thymoquinone-induced production of reactive oxygen species promotes dedifferentiation through the ERK pathway and inflammation through the p38 and PI3K pathways in rabbit articular chondrocytes
}

\author{
SEON-MI YU and SONG-JA KIM \\ Department of Biological Sciences, Kongju National University, Gongju 314-701, Republic of Korea
}

Received July 8, 2014; Accepted November 17, 2014

DOI: $10.3892 / \mathrm{ijmm} .2014 .2014$

\begin{abstract}
Dedifferentiation and inflammation are major features of cartilage degeneration during the pathogenesis of osteoarthritis (OA). Thymoquinone (TQ) is the major compound of black seed oil isolated from Nigella sativa with various beneficial or harmful effects on several diseases; however, its effects on the dedifferentiation and inflammation of chondrocytes have not yet been characterized. In the present study, we investigated whether TQ regulates the dedifferentiation and inflammation of rabbit articular chondrocytes, focusing on the production of reactive oxygen species (ROS) in rabbit articular chondrocytes. TQ induced the generation of ROS in a dose-dependent manner, as shown by staining with the fluorescent probe, 2'-7'-dichlorofluorescein diacetate. We confirmed that TQ induced dedifferentiation by measuring the loss of type II collagen and the reduction in chondroitin sulfate proteoglycan levels. TQ also caused inflammation by inducing the expression of cyclooxygenase-2 (COX-2) and prostaglandin $\mathrm{E}_{2}\left(\mathrm{PGE}_{2}\right)$. The antioxidant, $\mathrm{N}$-acetyl cysteine (NAC), prevented the dedifferentiation and inflammation which was generated by the TQ-induced production of ROS. Furthermore, TQ caused a dose-dependent increase in p38, phosphorylated extracellular signal-regulated kinase (p-ERK) and phosphoinositide 3-kinase (PI3K) expression. NAC abrogated this effect and attenuated the dedifferentiation and inflammation which was generated by the TQ-induced production of ROS. To identify the ROS-regulated pathways, we treated the chondrocytes with the p38 inhibitor, SB203580, the MEK inhibitor, PD98059, and the PI3K inhibitor, LY294002. PD98059 inhibited the TQ-induced dedifferentiation and
\end{abstract}

Correspondence to: Professor Song-Ja Kim, Department of Biological Sciences, Kongju National University, 56 Daehakro, Gongju 314-701, Republic of Korea

E-mail:ksj85@kongju.ac.kr

Key words: thymoquinone, chondrocytes, reactive oxygen species, dedifferentiation
SB203580 and LY294002 prevented the TQ-induced inflammation. These findings suggest that the TQ-induced production of ROS causes dedifferentiation through the ERK pathway and inflammation through the PI3K and p38 pathways in rabbit articular chondrocytes.

\section{Introduction}

Osteoarthritis (OA), a degenerative joint disease, is a multifactorial process in which mechanical factors play a central role and is characterized by alterations in the structure and function of the whole joint (1). OA involves the entire joint organ, including the subchondral bone, meniscus, ligaments, periarticular muscle, capsule and synovium, and is associated with risk factors, such as age, gender, obesity, prior joint injury, genetic predisposition and mechanical factors, including malalignment and abnormal joint shape (2). During skeletal development, chondrocytes differentiate from mesenchymal progenitors to synthesize the cartilage (3). Differentiated chondrocytes express cartilage-specific collagens II, IX and XI. Under normal conditions, chondrocytes rest in a non-stimulated steady state and maintain the synthesis of proteoglycans and other non-collagen molecules (4).

Prostaglandins are produced by cyclooxygenases (COX) from arachidonic acid and are induced in arthritic joints (5). COX has three forms: COX-1, COX-2 and COX-3. Whereas COX-1 is constituvely expressed in various cell types to maintain homeostasis, COX-2 is the inducible form of COX, implicated in prostaglandin synthesis in the inflammatory response and has been associated with osteoarthritic cartilage (6). COX-3 is a recently described variant of COX-1 and is also known as COX-1 VI. However, to date, there is not much conclusive evidence available regarding the existence of COX-3 protein (7).

Reactive oxygen species (ROS), such as hydrogen peroxide $\left(\mathrm{H}_{2} \mathrm{O}_{2}\right)$, superoxide anion $\left(\mathrm{O}_{2}{ }^{-}\right)$. and hydroxyl radical $\left({ }^{\circ} \mathrm{OH}\right)$ are generally believed to be harmful to cells and tissues (8). ROS are generated by a variety of endogenous and exogenous processes through several pathways and the mitochondria are the major source of intracellular $\operatorname{ROS}(9,10)$. ROS are destructive to DNA and proteins (11). ROS are involved in the regulation of the production of biochemical factors involved in 
cartilage degradation. They may cause damage to all matrix components, either directly or indirectly by reducing matrix component synthesis (12).

Thymoquinone (TQ) is the main active component of Nigella sativa oil, traditionally used in the Middle East (13). In this study, we investigated the effects of TQ and the regulatory mechanisms of TQ with respect to dedifferentiation and COX-2 expression in chondrocytes, including alterations in the expression of various signaling molecules in TQ-treated chondrocytes. Several signaling cascades, including those involving phosphoinositide 3-kinase (PI3K)/Akt and mitogenactivated protein kinases (MAPKs; p38, ERK) and c-Jun $\mathrm{N}$-terminal kinase (JNK), regulate the dedifferentiation of chondrocytes and COX-2 expression by modulating the generation of $\operatorname{ROS}(14,15)$. Although other investigators have suggested that ROS inhibit differentiation and induce COX-2 expression, the mechanisms involved have not been fully elucidated. In the present study, we investigated the molecular mechanisms through which the TQ-induced generation of ROS affects dedifferentiation and COX-2 expression in rabbit articular chondrocytes. Our results suggest that TQ induces the generation of ROS, which modulates the PI3K/Akt or MAPK signaling cascades, leading to dedifferentiation and inflammation in rabbit chondrocytes.

\section{Materials and methods}

Primary culture of rabbit articular chondrocytes. Articular chondrocytes were isolated from cartilage slices of 2-week-old New Zealand white rabbits (Koatech, Pyeongtaek, Korea), as previously described (16). Briefly, the cartilage slices were enzymatically dissociated in $0.2 \%$ collagenase in Dulbecco's modified Eagle's medium (DMEM; Gibco, Carlsbad, CA, USA). Individual cells were cultured in DMEM supplemented with $10 \%$ (v/v) fetal bovine calf serum (Gibco). The chondrocytes were grown at $37^{\circ} \mathrm{C}$ in the DMEM in a humidified incubator containing $5 \% \mathrm{CO}_{2}$. Primary chondrocyte cultures at 3.5 days were treated with $0.1 \%$ DMSO (vehicle control) or with various pharmacological reagents, including TQ (Sigma-Aldrich, St. Louis, MO, USA). The cells were treated with various inhibitors [N-acetyl-L-cysteine (NAC), 4,4'-diisothiocyano-2,2'-stilbenedisulphonic acid (DIDS), SP600125, SB203580, PD98059 and LY294002] for $1 \mathrm{~h}$ prior to treatment with TQ. NAC and DIDS were purchased from Sigma-Aldrich, and SP600125 was obtained from Biomol (Plymouth Meeting, PA, USA). The other chemicals used, SB203580 and PD98059, were purchased from Calbiochem (San Diego, CA, USA). LY294002 was obtained from Tocris Bioscience (Bristol, Avon, UK). The study was approved by the Ethics Committee of Kongju National University, Gongju, Korea.

Western blot analysis. The cells were lysed in radioimmunoprecipitation (RIPA) lysis buffer containing protease inhibitors [10 g/ml leupeptin, $10 \mathrm{~g} / \mathrm{ml}$ pepstatin A, $10 \mathrm{~g} / \mathrm{ml}$ aprotinin and $1 \mathrm{mM}$ 4-(2-aminoethyl)benzenesulfonyl fluoride] and phosphatase inhibitors $\left(1 \mathrm{mM} \mathrm{NaF}\right.$ and $\left.1 \mathrm{mM} \mathrm{Na} \mathrm{VO}_{4}\right)$. Protease inhibitors and phosphatase inhibitors were obtained from Sigma-Aldrich. Equal amounts of protein were mixed with electrophoresis sample buffer (Bio-Rad Laboratories, Hercules, CA, USA) and boiled for $5 \mathrm{~min}$ before loading onto SDS-PAGE gels. Proteins were fractionated by SDS-PAGE and transferred onto nitrocellulose membranes (Millipore, Billerica, MA, USA). The membranes were incubated with primary antibodies followed by horseradish peroxidase-conjugated secondary antibodies (Sigma-Aldrich). Primary antibodies were specific to phosphorylated (p-)p38 (\#9211; Cell Signaling Technology, Beverly, MA, USA), p-ERK-1/2 (\#9101; Cell Signaling Technology), p-JNK (\#9251; Cell Signaling Technology), p-Akt (\#9271; Cell Signaling Technology), type II collagen (MAB8887; Santa Cruz Biotechnology, Santa Cruz, CA, USA), actin (sc-1615; Santa Cruz Biotechnology) and COX-2 (\#160106; Cayman Chemical Co., Ann Arbor, MI, USA). Proteins were visualized with ECL Plus reagent (Amersham Biosciences) on a Chemilumino analyzer LAS 4000 mini (Fujifilm, Tokyo, Japan).

Chondrocyte differentiation. The chondrocytes were identified by staining for sulfate proteoglycan with Alcian blue as previously described (17). The cells were washed twice with cold PBS, fixed with $95 \%$ methanol for $2 \min \left(-20^{\circ} \mathrm{C}\right)$ and stained overnight with $0.1 \%$ Alcian Blue 8GX (Wako Pure Chemical Industries Ltd., Osaka, Japan) in $0.1 \mathrm{M} \mathrm{HCl}$. After washing 3 times with distilled water, the stain was extracted with $800 \mu \mathrm{l}$ of $6 \mathrm{M}$ guanidine- $\mathrm{HCl}$ for $6 \mathrm{~h}$ at room temperature; optical density was measured at $595 \mathrm{~nm}$.

Measurement of ROS production. The fluorogenic marker, 7'-dichlorodihydrofluorescein diacetate (DCFH-DA; Sigma-Aldrich), was used to monitor the production of intracellular ROS. Following treatment with various concentrations of TQ for $2 \mathrm{~h}$, the cells were washed twice with PBS and loaded for 30 min with DCFH-DA (10 $\mu \mathrm{M}$; Sigma-Aldrich) in DMEM without phenol red. The acetoxymethyl group on DCFH-DA is cleaved by non-specific esterases within the cell, producing a non-fluorescent charged molecule that does not cross the cell membrane. Intracellular ROS irreversibly oxidizes DCFH-DA to dichlorofluorescein (DCF), which is a fluorescent product. Following treatment, the medium was removed, the chondrocytes were collected by centrifugation and fluorescence was measured on an Flx8000 fluorometer (excitation, $485 \mathrm{~nm} /$ emission, $525 \mathrm{~nm}$; Bio-Tek Instruments, Winooski, VT, USA). For ROS visualization by fluorescence microscopy, the cells were labeled for $30 \mathrm{~min}$ at $37^{\circ} \mathrm{C}$ in the dark with DCFH-DA $(10 \mu \mathrm{M})$ probe. The chondrocytes were washed twice with PBS. Fluorescence was observed under an inverted Olympus BX50 microscope (Olympus, Tokyo, Japan). DCF fluorescence intensity was quantified using ImageJ software (Vector Laboratories, Burlingame, CA, USA).

Immunofluorescence (IF) staining. The chondrocytes cultured on glass coverslips were fixed in $4 \%$ paraformaldehyde at $4{ }^{\circ} \mathrm{C}$ for $10 \mathrm{~min}$ and permeabilized with $0.1 \%$ Tween-20 in PBS for $15 \mathrm{~min}$. For immunostaining, goat polyclonal antibody to type II collagen (MAB8887; 1:50 dilution; Santa Cruz Biotechnology) and anti-rabbit polyclonal antibody to COX-2 (\#160112; 1:50 dilution; Cayman Chemical Co.) were used as primary and secondary antibodies, respectively. Counterstaining with DAPI (Molecular Probe) enabled nuclear visualization. Images of the cultured chondrocytes were acquired using a fluorescence microscope (Olympus BX50; Olympus). 
A
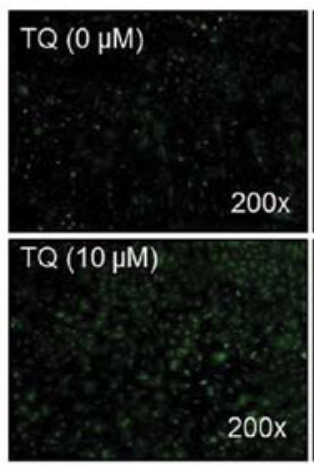
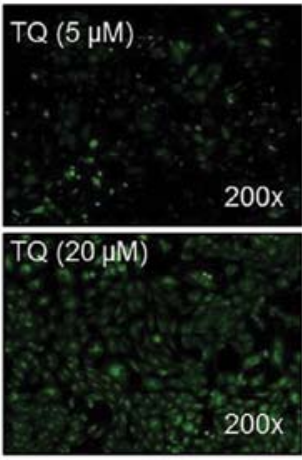

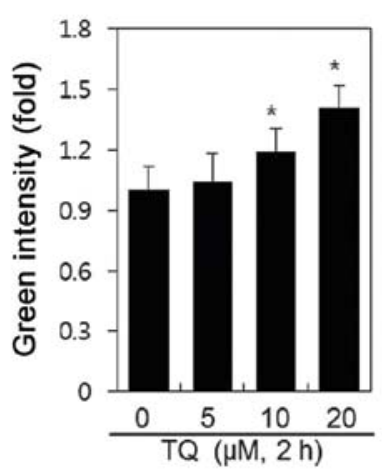

B

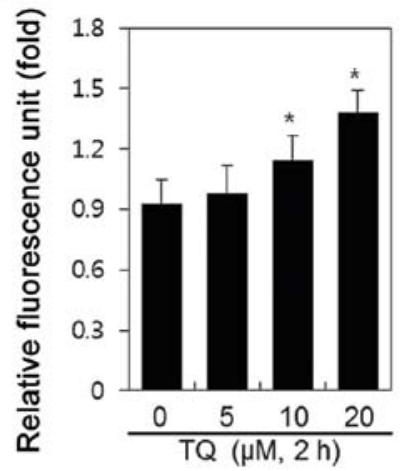

Figure 1. Thymoquinone (TQ) induces the generation of intracellular reactive oxygen species (ROS) in primary rabbit articular chondrocytes. (A) Chondrocytes were exposed to TQ for $2 \mathrm{~h}$. Dichlorofluorescein (DCF) fluorescence intensity was observed under an inverted fluorescence microscopy (left panel). DCF fluorescence intensity was quantified using ImageJ software (right panel). (B) Chondrocytes were exposed to TQ for $2 \mathrm{~h}$. ROS fluorescence was measured using an Flx8000 Bio-Tek fluorometer (B). Data are presented as the means \pm SD from 3 independent experiments performed in triplicate. ${ }^{*}<<0.05$, compared with the control group.

Measurement of prostaglandin $E_{2}\left(P G E_{2}\right)$ levels. The chondrocytes were seeded in standard 96-well microtiter plates at $1 \times 10^{4}$ cells/well. Following treatment, COX-2 activity was determined by measuring $\mathrm{PGE}_{2}$ levels in the culture medium. $\mathrm{PGE}_{2}$ concentrations were determined using a standardized enzyme immunoassay (EIA) according to the manufacturer's instructions (Assay Designs, Ann Arbor, MI, USA).

Statistical analysis. Data are expressed as the means \pm SEM and analyzed by one-way analysis of variance (ANOVA). Comparisons between groups were performed by ANOVA followed by Turkey's multiple comparison, comparing all groups to the DMSO-treated group (control). Graphs were generated using Microsoft Excel 2007. P-values <0.05 were considered to indicate statistically significant differences.

\section{Results}

To the best of our knowledge, this is the first study assessing the effects of TQ on normal rabbit articular chondrocytes. Chondrocytes were treated with TQ $(0,5,10$ and $20 \mu \mathrm{M})$ for $2 \mathrm{~h}$, after which we observed a marked induction of ROS generation by fluorescence microscopy (Fig. 1A) and fluorometry (Fig. 1B). The dose-dependent increase in the production of ROS increased 1.4-fold after $2 \mathrm{~h}$ of treatment with TQ, as shown in Fig. 1B. These results indicate that TQ induces ROS production in rabbit articular chondrocytes.

To determine whether TQ influences the chondrocyte phenotype, the cells were treated with various concentrations of TQ for $24 \mathrm{~h}$ or with $20 \mu \mathrm{M}$ of TQ for various periods of time (Fig. 2). The production of type II collagen, a differentiation marker, was inhibited in a dose- and time-dependent manner (Fig. 2A and B) following treatment with TQ. Thus, TQ is capable of inducing the dedifferentiation of chondrocytes.

We also examined the effects of TQ on the production of chondroitin sulfate proteoglycan, which accumulates during chondrocyte differentiation. The TQ-treated chondrocytes exhibited a dose-dependent decrease in sulfate proteoglycan staining in comparison to the controls (Fig. 2C). IF staining revealed that type II collagen was distributed throughout the extracellular matrix of the control cells. However, the type II collagen levels were decreased in the TQ-treated chondrocytes (Fig. 2D). These findings suggest that TQ induces the dedifferentiation of rabbit articular chondrocytes.

We sought to determine whether TQ affects COX-2 expression. The chondrocytes were treated with various concentrations of TQ for $24 \mathrm{~h}$ or with $20 \mu \mathrm{M}$ of TQ for various periods of time (Fig. 3). The expression of the inflammatory mediator, COX-2, was induced in a dose- and time-dependent manner (Fig. 3A and B) following treatment with TQ. We also found that TQ induced a marked dose-dependent induction in $\mathrm{PGE}_{2}$ synthesis, which is known to mediate inflammation (Fig. 3C). IF staining revealed that COX-2 was distributed in low amounts throughout the cytosol of the control cells; however, COX-2 expression was greater in the TQ-treated chondrocytes (Fig. 3D). These data suggest that TQ induces COX-2 expression in rabbit articular chondrocytes.

After observing the prominent effects of TQ on dedifferentiation and COX-2 expression in chondrocytes, we sought to elucidate the mechanisms responsible for these effects. The effects of TQ on dedifferentiation and COX-2 expression in chondrocytes were determined after $24 \mathrm{~h}$ of treatment with $20 \mu \mathrm{M}$ TQ. ROS play a key role in dedifferentiation and inflammation $(9,18)$. Thus, ROS function as critical signaling molecules in various cell types, including chondrocytes.

TQ induced the production of ROS (Fig. 4A); thus, this production of ROS may mediate the TQ-induced dedifferentiation and expression of COX-2 in chondrocytes. We examined this hypothesis by assessing the effects of TQ in the presence of NAC, a ROS scavenger (Fig. 4). The TQ-treated chondrocytes were incubated with NAC $(5 \mathrm{mM})$ for $24 \mathrm{~h}$ and then analyzed by fluorescence microscopy (Fig. 4A). In the TQ-treated cells, a 1.5-fold increase in ROS production was observed; no change was observed in ROS production in the control cells. Treatment of the chondrocytes with TQ in the presence of NAC resulted in only a 1.1-fold increase in ROS production (Fig. 4B). Blocking the generation of ROS with NAC nearly abolished the TQ-induced loss of type II collagen, as well as the increase 


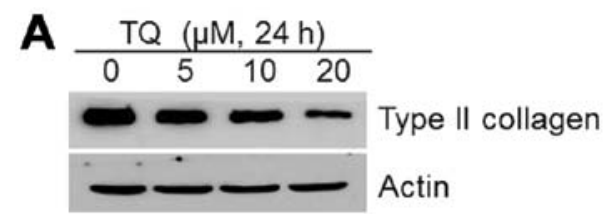

C

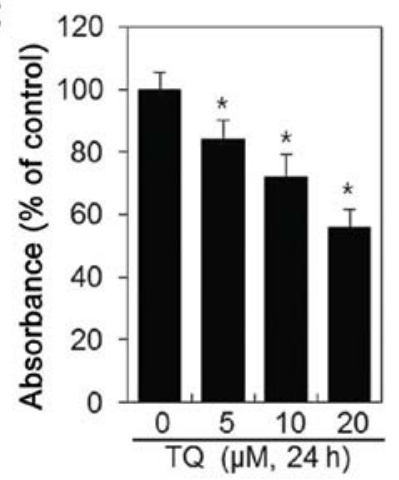

B

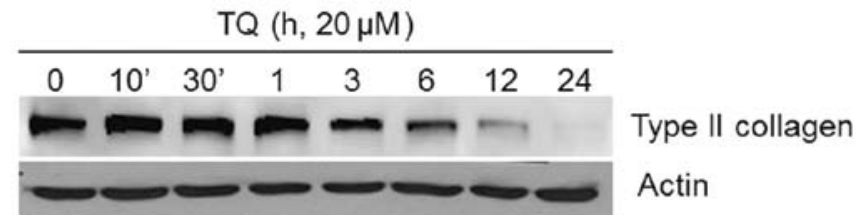

D

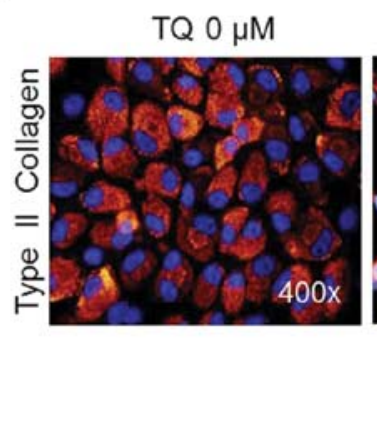

TQ $20 \mu \mathrm{M}$

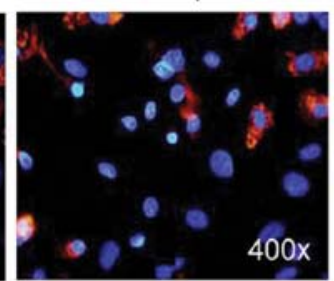

Figure 2. Thymoquinone (TQ) induces dedifferentiation in rabbit articular chondrocytes. (A) Chondrocytes were exposed to TQ for $24 \mathrm{~h}$. (B) Cells were exposed to $20 \mu \mathrm{M}$ TQ for $24 \mathrm{~h}$. (A and B) The expression of type II collagen and actin was determined by western blot analysis with actin as a loading control. Chondrocytes were treated with TQ for $24 \mathrm{~h}$. (C) The synthesis of sulfate proteoglycan was determined by Alcian blue staining. Articular chondrocytes were exposed to $20 \mu \mathrm{M}$ TQ for $24 \mathrm{~h}$. (D) The expression of type II collagen was determined by IF staining. Data are presented as the means \pm SD from 3 independent experiments performed in triplicate. ${ }^{*} \mathrm{P}<0.05$, compared with the control group.
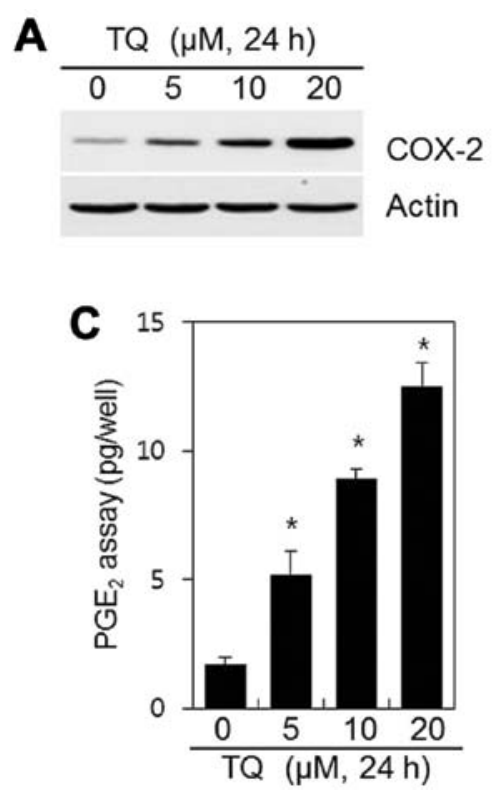

B

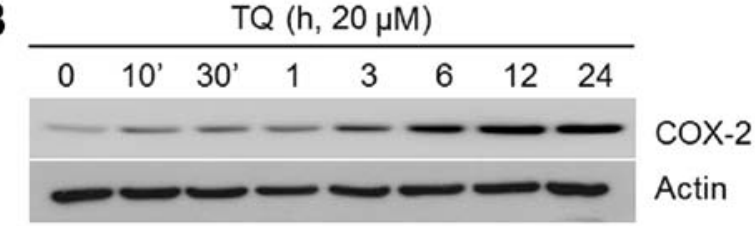

D

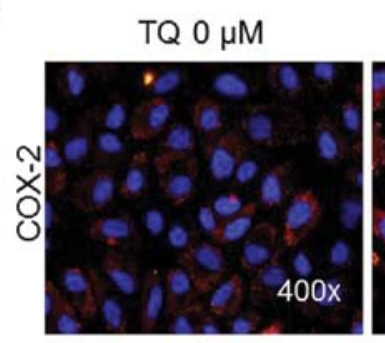

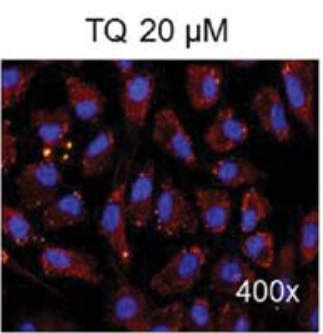

Figure 3. Thymoquinone (TQ) increases cyclooxygenase-2 (COX-2) expression in rabbit articular chondrocytes. (A) Primary chondrocytes were exposed to TQ for $24 \mathrm{~h}$. (B) Cells were exposed to $20 \mu \mathrm{M}$ TQ for $24 \mathrm{~h}$. (A and B) The expression of COX-2 and actin was determined by western blot analysis with actin as a loading control. (C) Rabbit chondrocytes were treated with TQ for $24 \mathrm{~h}$. (C) Production of prostaglandin $\mathrm{E}_{2}\left(\mathrm{PGE}_{2}\right)$ was determined by $\mathrm{PGE}_{2}$ assay. (D) Chondrocytes were exposed to $20 \mu \mathrm{M}$ TQ for $24 \mathrm{~h}$. (D) The expression of COX-2 was determined by immunofluorescence staining. Data are presented as the means \pm SD from 3 independent experiments performed in triplicate. ${ }^{*}<<0.05$, compared with the control group.

in $\mathrm{COX}-2$ expression and $\mathrm{PGE}_{2}$ production in chondrocytes (Fig. 4C-E).

In order to gain further insight into the molecular mechanisms underlying the induction of differentiation and COX-2 expression in chondrocytes, we investigated the activation of the MAPK and PI3K pathways (Fig. 5). Our results revealed that TQ induced a dose-dependent increase in the expres- sion of the MAPKs, p-p38, p-ERK, p-JNK and PI3K/pAkt (Fig. 5A). The TQ-induced phosphorylation of MAPKs and PI3K was long-lasting and reached maximum levels after 10 min of treatment for p-p38, p-ERK and p-JNK and $30 \mathrm{~min}$ for p-Akt; the levels decreased thereafter (Fig. 5B). We then determined whether the TQ-induced activation of MAPKs and PI3K is blocked by NAC (Fig. 6A). NAC inhibited the 
A
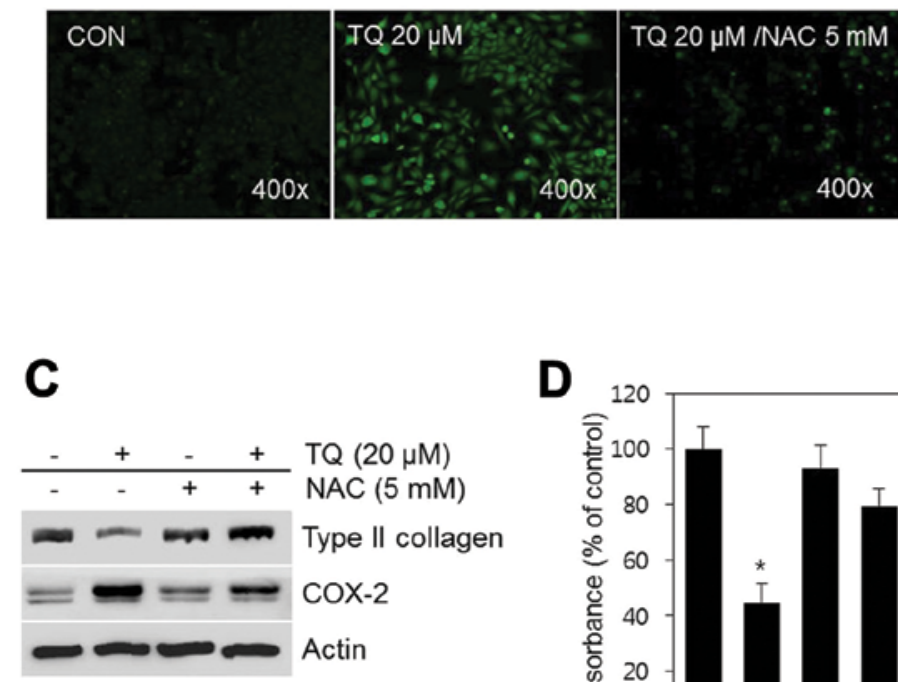

B

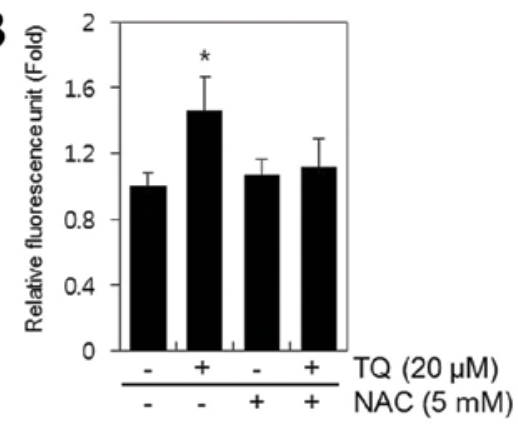

$\mathbf{E}$

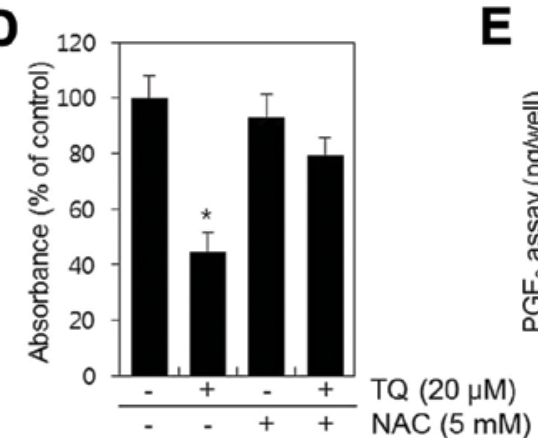

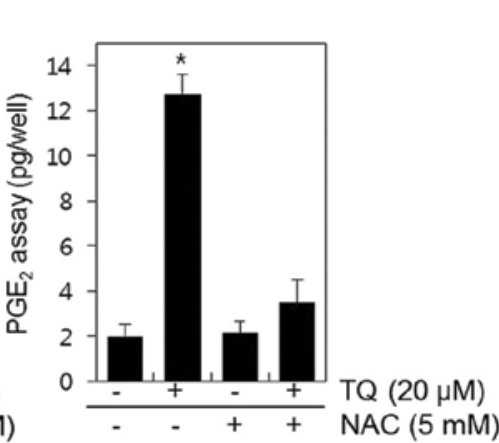

Figure 4. Thymoquinone (TQ)-induced dedifferentiation and cyclooxygenase-2 (COX-2) expression are blocked by an inhibitor of reactive oxygen species (ROS), N-acetyl cysteine (NAC). Chondrocytes were exposed to $20 \mu \mathrm{M}$ TQ in the absence or presence of $5 \mathrm{mM} \mathrm{NAC} \mathrm{(A} \mathrm{and} \mathrm{B)} \mathrm{for} 2 \mathrm{~h}$ or (C and D) for $24 \mathrm{~h}$. ROS production was determined by fluorescence microscopy (A; magnification, x200). (B) Reactive oxygen species (ROS) fluorescence was measured using an Flx8000 fluorometer. (C) The expression of type II collagen and COX-2 was determined by western blot analysis with actin as a loading control. (D) The synthesis of sulfate proteoglycan was detected by Alcian blue staining. (E) The secretion of prostaglandin $\mathrm{E}_{2}\left(\mathrm{PGE}_{2}\right)$ was analyzed by $\mathrm{PGE}_{2}$ assay. Data are presented as the means \pm SD from 3 independent experiments performed in triplicate. " $\mathrm{P}<0.01$, compared with the control group.
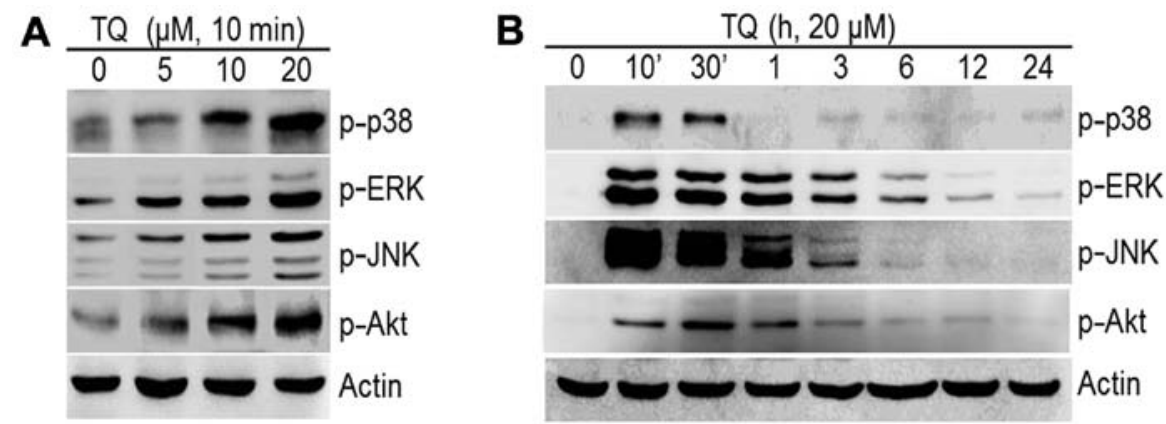

Figure 5. Thymoquinone (TQ) activates the PI3K/Akt and the MAPK (p38, ERK and JNK) pathways in chondrocytes. (A) Rabbit chondrocytes were exposed to TQ for $24 \mathrm{~h}$. (B) Articular chondrocytes were exposed to $20 \mu \mathrm{M}$ TQ for the indicated periods of time. (A and B) The activation of p38, ERK, JNK and Akt was determined by western blot analysis with actin as a loading control. Data are presented as the means \pm SD from 3 independent experiments performed in triplicate.

TQ-induced phosphorylation of MAPKs and PI3K (Fig. 6A). To determine the association between the TQ-induced generation of ROS, dedifferentiation and COX-2 expression and the activation of MAPKs and PI3K, we inhibited the phosphorylation of MAPKs and PI3K using specific inhibitors (SB203580 for p38, PD98059 for ERK, LY294002 for PI3K/Akt and SP600125 for JNK) prior to treatment with TQ (Fig. 6). None of these inhibitors blocked the TQ-induced generation of ROS, but some slightly inhibited the TQ-induced dedifferentiation and the expression of COX-2 (Fig. 6B and C). The inhibition of ERK by PD98059 attenuated the TQ-induced loss in type II collagen expression and proteoglycan synthesis (Fig. 6B and C). The inhibition of p38 with SB203580 or PI3K/Akt with
LY294002 blocked the TQ-induced expression of COX-2 and $\mathrm{PGE}_{2}$ synthesis (Fig. 6C and E).

A recent study demonstrated that DIDS, a selective inhibitor of mitochondrial electron transport, prevents the production of ROS (19). In the present study, the cells treated with DIDS no longer produced ROS in response to TQ (Fig. 7A). Treatment with DIDS restored type II collagen expression and sulfate proteoglycan synthesis and decreased the expression of COX-2 and $\mathrm{PGE}_{2}$ production in the TQ-treated chondrocytes (Fig. 7B-D). These findings suggest that the inhibition of ROS production from the mitochondria by DIDS inhibits dedifferentiation and COX-2 expression and that ROS is the key source of cartilage destruction (Fig. 7). 

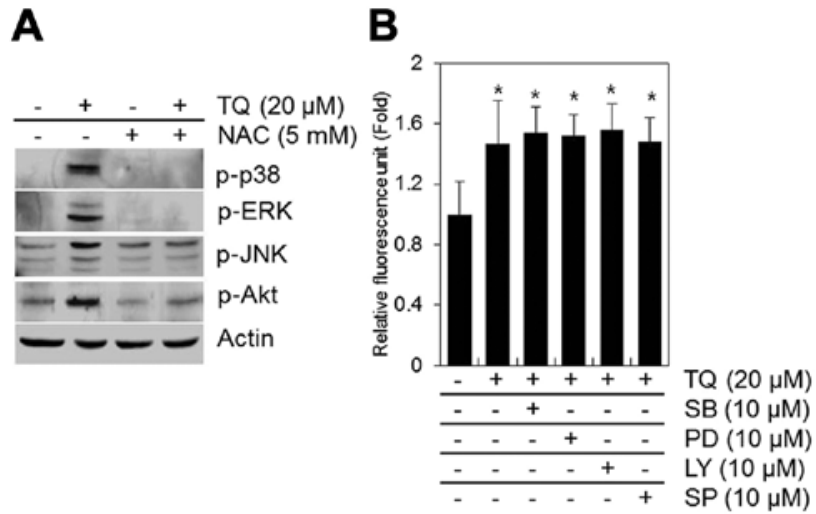

C

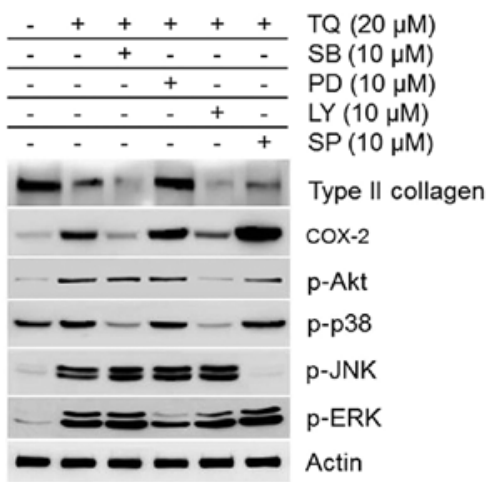

D

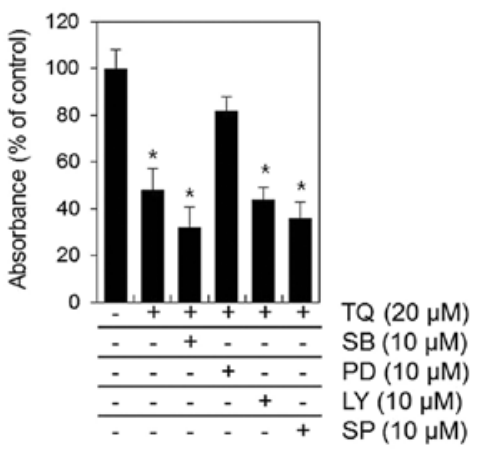

E

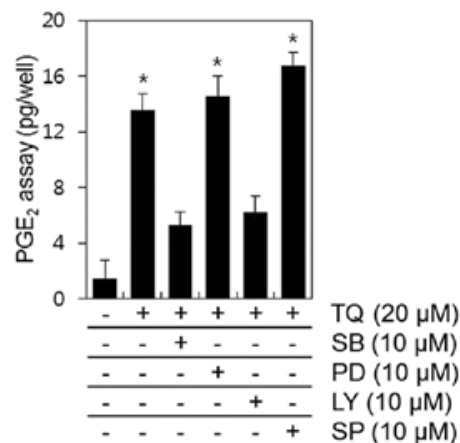

Figure 6. Thymoquinone (TQ)-induced reactive oxygen species (ROS) generation regulates dedifferentiation through p38 and cyclooxygenase-2 (COX-2) expression through PI3K/Akt and ERK. (A) Chondrocytes were exposed to $20 \mu \mathrm{M}$ TQ in the absence or presence of $5 \mathrm{mM} \mathrm{N}$-acetyl cysteine (NAC) for $24 \mathrm{~h}$. (A) The expression of p-p38, p-ERK, p-JNK, p-Akt and actin was determined by western blot analysis with actin as a loading control. Primary chondrocytes were exposed to $20 \mu \mathrm{M}$ TQ in the absence or presence of SB203580 (SB, PI3K inhibitor), PD98059 (PD, p38 inhibitor), LY294002 (LY, ERK inhibitor) or SP600125 (SP, JNK inhibitor) (B) for $2 \mathrm{~h}$ or (C-E) for $24 \mathrm{~h}$. (B) ROS fluorescence was measured using an Flx 8000 fluorometer. (C) The expression of type II collagen, COX-2, p-Akt, p-p38, p-JNK and p-ERK was determined by western blot analysis with actin as a loading control. (D) The production of sulfate proteoglycan was determined by Alcian blue staining. (E) The synthesis of prostaglandin $\mathrm{E}_{2}\left(\mathrm{PGE}_{2}\right)$ was analyzed by $\mathrm{PGE}_{2}$ assay. Data are presented as the means \pm SD from 3 independent experiments performed in triplicate. ${ }^{*} \mathrm{P}<0.01$, compared with the control group.

A

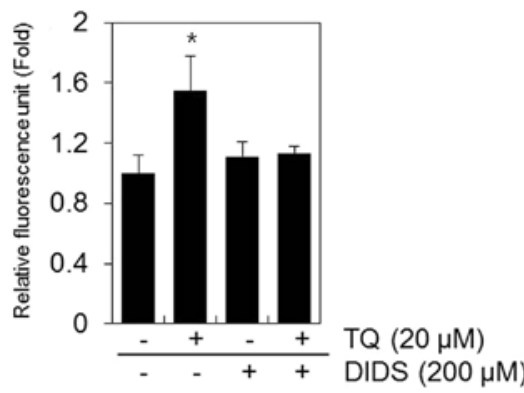

B

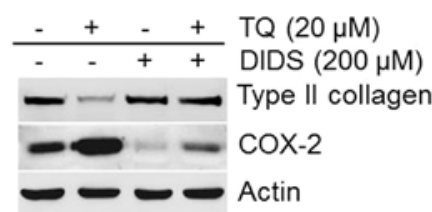

C

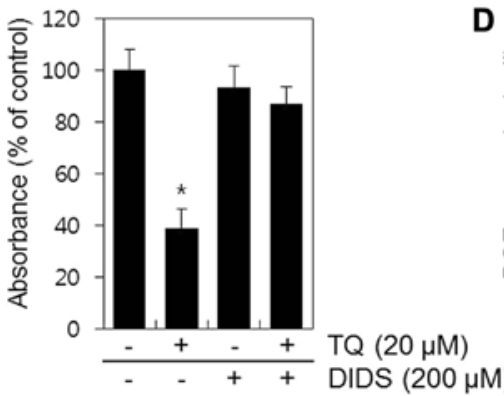

D

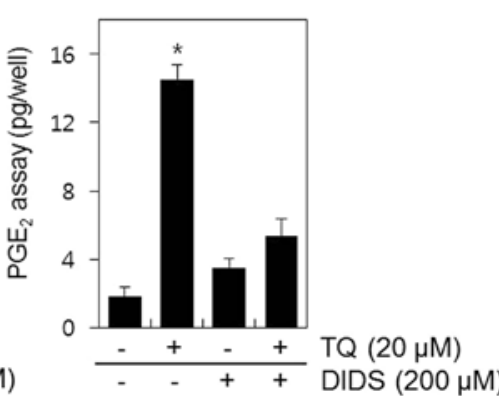

Figure 7. 4,4'-Diisothiocyano-2,2'-stilbenedisulphonic acid (DIDS) inhibits dedifferentiation and cyclooxygenase-2 (COX-2) expression which is generated by the thymoquinone (TQ)-induced production of reactive oxygen species (ROS). Chondrocytes were exposed to $20 \mu \mathrm{M} \mathrm{TQ}$ in the presence or absence of $200 \mu \mathrm{M}$ DIDS for (A) $2 \mathrm{~h}$ or (B-D) $24 \mathrm{~h}$. (A) ROS fluorescence was measured using an Flx8000 fluorometer. (B) The expression of type II collagen and COX-2 was determined by western blot analysis with actin as a loading control. (C) The production of sulfate proteoglycan was determined by Alcian blue staining. (E) The synthesis of prostaglandin $\mathrm{E}_{2}\left(\mathrm{PGE}_{2}\right)$ was analyzed by $\mathrm{PGE}_{2}$ assay. Data are presented as the means $\pm \mathrm{SD}$ from 3 independent experiments performed in triplicate. ${ }^{*} \mathrm{P}<0.05$, compared with the control group. 


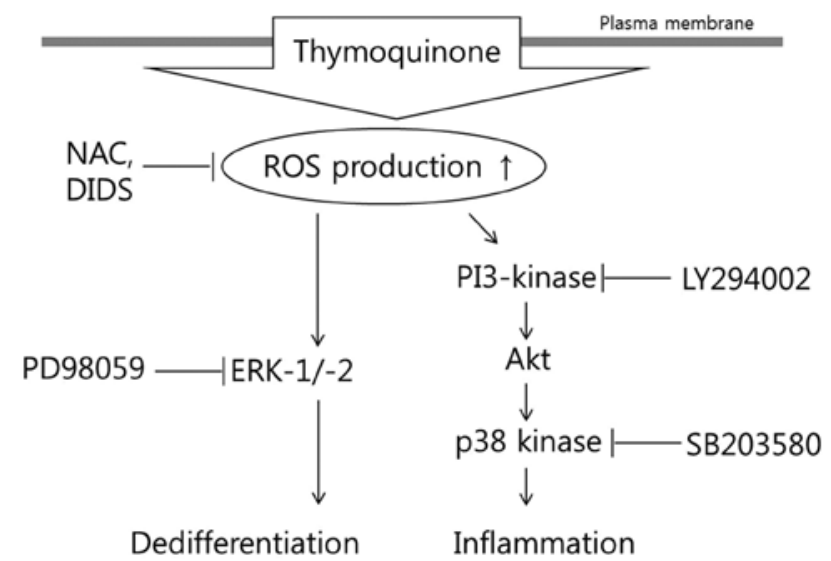

Figure 8. Schematic diagram of thymoquinone (TQ)-induced dedifferentiation and cyclooxygenase-2 (COX-2) expression. The TQ-induced production of reactive oxygen species (ROS) regulates dedifferentiation through the ERK pathway and modulates COX-2 expression through the PI3K and p38 pathways. These effects are blocked by treatment with N-acetyl cysteine (NAC) and 4,4'-diisothiocyano-2,2'-stilbenedisulphonic acid (DIDS).

A schematic diagram displaying the cascade of TQ -induced dedifferentiation and inflammation and the mechanisms involved is presented in Fig. 8.

\section{Discussion}

The pathogenesis of $\mathrm{OA}$ is associated with risk factors, such as oxidative stress and free radicals $(20,21)$. Oxidative stress is caused by abnormal cell metabolism exceeding the physiologicalbuffering capacity. Oxidative stress has been described to increase cellular aging, thus weakening organ function (22). Previous studies have demonstrated that OA cartilage has high oxidative activity $(23,24)$. ROS overproduction in cartilage originates in the mitochondria and results in chondrocyte destruction, which in turn causes OA (25). ROS serve as second messengers that mediate gene transcription, cell proliferation, necrosis, apoptosis and differentiation in a variety of cell types (26). In this study, we found that TQ induced intracellular ROS production and induced dedifferentiation and COX-2 expression in chondrocytes. TQ induced a dose-dependent increase in ROS production (Fig. 1). TQ also induced the loss of type II collagen and an increase in COX-2 expression (Figs. 1 and 2), while NAC inhibited the TQ-induced dedifferentiation and inflammation (Fig. 3). These findings demonstrate that TQ is an effective inducer of ROS generation in chondrocytes, indicating that TQ may play a role in the process of cartilage destruction through ROS-mediated pathways. Since treatment of the chondrocytes with low concentrations of TQ $(<5 \mu \mathrm{M})$ than those used in the present study had no effect on ROS accumulation, type II collagen and COX-2 expression (data not shown), we used concentrations of $5-20 \mu \mathrm{M}$ in this study. In our previous study, we demonstrated that the treatment of chondrocytes with TQ (5-20 $\mu \mathrm{M})$ resulted in apoptosis, suggesting that TQ may be effectively used to elucidate the pathways or mechanisms responsible for apoptosis in chondrocytes (27). TQ may thus be a suitable reagent for determining the mechanisms responsible for dedifferentiation and inflammation.
Increasing evidence has attributed cellular damage in a variety of disorders in humans to oxidative stress that leads to ROS production, and these effects are mediated by the interaction with matrix metalloproteinases (MMPs) $(28,29)$. Therefore, in this study, we investigated whether ROS leads to the destruction of matrix components, such as type II collagen, by activating MMPs. However, our results indicated that TQ did not affect MMP production (data not shown).

Our findings also suggested that TQ increased the expression and production of the pro-inflammatory mediators, COX-2 and $\mathrm{PGE}_{2}$ (Fig. 3). Dedifferentiation and inflammation are supported by an intracellular signaling network involving the PI3K/Akt and MAPKs pathways (30). Phosphorated Akt translocates to the nucleus and phosphorylates numerous target molecules to mediate signals (31). MAPKs are a family of proteins promoting a phosphorylative signaling cascade, leading to the activation of transcription factors involved either in cellular dedifferentiation and inflammation (32). It has also been reported that ROS induces dedifferentiation, inflammation and proliferation in a variety of cell types through the temporal activation of the PI3K and MAPKs pathways $(31,32)$. In addition, several studies have linked dedifferentiation and COX-2 expression with MAPKs, p38, ERK-1/2 and JNK and PI3K/Akt. $(21,33,34)$.

In the present study, TQ induced the activation of MAPKs and PI3K (Fig. 5) and the inhibition of TQ-induced dedifferentiation by PD98059 was due to the inhibition of ERK activation (Fig. 6C). The inhibition of p38 and PI3K decreased the TQ-induced expression of COX-2, but did not influence dedifferentiation (Fig. 6C).

As demonstrated in our study, DIDS inhibits anion channels in the mitochondrial inner membrane, thus, inhibiting ROS release from the organelle. Pre-treatment of the TQ-treated cells with DIDS abolished dedifferentiation and COX-2 expression, suggesting that the transition of ROS through anion channels may be required for the activation of the MAPK and PI3K pathways (Fig. 7). Thus, our results indicate that the TQ-induced production of ROS triggers dedifferentiation through ERK and COX-2 expression through the p38 and PI3K pathways.

\section{Acknowledgements}

The present study was supported by the Korean Health Technology R\&D Project, Ministry of Health and Welfare, Republic of Korea (A120960-1201-0000300) and the National Research Foundation of Korea (NRF) (MEST) (NRF-2012R1A1A2043276).

\section{References}

1. Hunter DJ and Felson DT: Osteoarthritis. BMJ 332: 639-642, 2006.

2. Blagojevic M, Jinks C, Jeffery A and Jordan KP: Risk factors for onset of osteoarthritis of the knee in older adults: a systematic review and meta-analysis. Osteoarthritis Cartilage 18: 24-33, 2010.

3. Goldring MB, Tsuchimochi K and Ijiri K: The control of chondrogenesis. J Cell Biochem 97: 33-44, 2006.

4. Maroudas A, Bayliss MT, Uchitel-Kaushansky N, Schneiderman R and Gilav E: Aggrecan turnover in human articular cartilage: use of aspartic acid racemization as a marker of molecular age. Arch Biochem Biophys 350: 61-71, 1998. 
5. Thomas B, Thirion S, Humbert L, et al: Differentiation regulates interleukin-1beta-induced cyclo-oxygenase-2 in human articular chondrocytes: role of p38 mitogen-activated protein kinase. Biochem J 362: 367-373, 2002.

6. Crofford LJ, Lipsky PE, Brooks P, Abramson SB, Simon LS and van de Putte LB: Basic biology and clinical application of specific cyclooxygenase-2 inhibitors. Arthritis Rheum 43: 4-13, 2000.

7. Burdan F, Chalas A and Szumilo J: Cyclooxygenase and prostanoids - biological implications. Postepy Hig Med Dosw (Online) 60: 129-141, 2006 (In Polish).

8. Droge W: Free radicals in the physiological control of cell function. Physiol Rev 82: 47-95, 2002.

9. Tormos KV, Anso E, Hamanaka RB, et al: Mitochondrial complex III ROS regulate adipocyte differentiation. Cell Metab 14: 537-544, 2011.

10. Nathan $C$ and Cunningham-Bussel A: Beyond oxidative stress: an immunologist's guide to reactive oxygen species. Nat Rev Immunol 13: 349-361, 2013.

11. Cadet J and Wagner JR: DNA base damage by reactive oxygen species, oxidizing agents, and UV radiation. Cold Spring Harb Perspect Biol 5: pii: a012559, 2013.

12. Sasaki K, Hattori T, Fujisawa T, Takahashi K, Inoue $H$ and Takigawa M: Nitric oxide mediates interleukin-1-induced gene expression of matrix metalloproteinases and basic fibroblast growth factor in cultured rabbit articular chondrocytes. J Biochem 123: 431-439, 1998

13. Woo CC, Kumar AP, Sethi G and Tan KH: Thymoquinone: potential cure for inflammatory disorders and cancer. Biochem Pharmacol 83: 443-451, 2012.

14. Kim KJ, Lee OH and Lee BY: Low-molecular-weight fucoidan regulates myogenic differentiation through the mitogen-activated protein kinase pathway in C2C12 cells. Br J Nutr 106: 1836-1844, 2011.

15. Wang $\mathrm{H}, \mathrm{Xi} \mathrm{S}, \mathrm{Xu} \mathrm{Y}$, et al: Sodium arsenite induces cyclooxygenase-2 expression in human uroepithelial cells through MAPK pathway activation and reactive oxygen species induction. Toxicol In Vitro 27: 1043-1048, 2013.

16. Yoon YM, Kim SJ, Oh CD, et al: Maintenance of differentiated phenotype of articular chondrocytes by protein kinase $\mathrm{C}$ and extracellular signal-regulated protein kinase. J Biol Chem 277: 8412-8420, 2002.

17. Asahina I, Sampath TK and Hauschka PV: Human osteogenic protein-1 induces chondroblastic, osteoblastic, and/or adipocytic differentiation of clonal murine target cells. Exp Cell Res 222 38-47, 1996.

18. Qi Z, Yin F, Lu L, et al: Baicalein reduces lipopolysaccharideinduced inflammation via suppressing JAK/STATs activation and ROS production. Inflamm Res 62: 845-855, 2013.

19. Pamenter ME, Ali SS, Tang Q, et al: An in vitro ischemic penumbral mimic perfusate increases NADPH oxidase-mediated superoxide production in cultured hippocampal neurons. Brain Res 1452: 165-172, 2012.

20. Dycus DL, Au AY, Grzanna MW, Wardlaw JL and Frondoza CG: Modulation of inflammation and oxidative stress in canine chondrocytes. Am J Vet Res 74: 983-989, 2013.
21. Yu SM and Kim SJ: Production of reactive oxygen species by withaferin A causes loss of type collagen expression and COX-2 expression through the PI3K/Akt, p38, and JNK pathways in rabbit articular chondrocytes. Exp Cell Res 319: 2822-2834, 2013.

22. Brouilette S, Singh RK, Thompson JR, Goodall AH and Samani NJ: White cell telomere length and risk of premature myocardial infarction. Arterioscler Thromb Vasc Biol 23: 842-846, 2003.

23. Bubici C, Papa S, Dean K and Franzoso G: Mutual cross-talk between reactive oxygen species and nuclear factor-kappa $\mathrm{B}$ : molecular basis and biological significance. Oncogene 25: 6731-6748, 2006

24. Tiku ML, Shah R and Allison GT: Evidence linking chondrocyte lipid peroxidation to cartilage matrix protein degradation. Possible role in cartilage aging and the pathogenesis of osteoarthritis. J Biol Chem 275: 20069-20076, 2000

25. Sauter E, Buckwalter JA, McKinley TO and Martin JA: Cytoskeletal dissolution blocks oxidant release and cell death in injured cartilage. J Orthop Res 30: 593-598, 2012.

26. Korbecki J, Baranowska-Bosiacka I, Gutowska I and Chlubek D: The effect of reactive oxygen species on the synthesis of prostanoids from arachidonic acid. J Physiol Pharmacol 64: 409-421, 2013.

27. Yu SM and Kim SJ: Thymoquinone-induced reactive oxygen species causes apoptosis of chondrocytes via PI3K/Akt and p38kinase pathway. Exp Biol Med (Maywood) 238: 811-820, 2013.

28. Kim MJ, Nepal S, Lee ES, Jeong TC, Kim SH and Park PH: Ethanol increases matrix metalloproteinase-12 expression via NADPH oxidase-dependent ROS production in macrophages. Toxicol Appl Pharmacol 273: 77-89, 2013.

29. Sawicki G: Synergistic effect of inhibitors of MMPs and ROS-dependent modifications of contractile proteins on protection hearts subjected to oxidative stress. Curr Pharm Des 20: 1345-1348, 2013

30. Torres M and Forman HJ: Redox signaling and the MAP kinase pathways. Biofactors 17: 287-296, 2003.

31. Wang X, Liu JZ, Hu JX, et al: ROS-activated p38 MAPK/ ERK-Akt cascade plays a central role in palmitic acid-stimulated hepatocyte proliferation. Free Radic Biol Med 51: 539-551, 2011.

32. Lin CF, Young KC, Bai CH, et al: Blockade of reactive oxygen species and Akt activation is critical for anti-inflammation and growth inhibition of metformin in phosphatase and tensin homolog-deficient RAW264.7 cells. Immunopharmacol Immunotoxicol 35: 669-677, 2013.

33. Eo SH, Cho HS and Kim SJ: Resveratrol regulates type II collagen and COX-2 expression via the ERK, p38 and Akt signaling pathways in rabbit articular chondrocytes. Exp Ther Med 7: 640-648, 2014.

34. Lee WK, Chung KW, Kim GH and Kim SJ: Gallotannin causes differentiation and inflammation via ERK1/2 and p38 kinase pathways in rabbit articular chondrocytes. Mol Med Rep 7: 701-707, 2013. 